\title{
CenH3 evolution in diploids and polyploids of three angiosperm genera
}

\author{
Rick E Masonbrink, Joseph P Gallagher, Josef J Jareczek, Simon Renny-Byfield, Corrinne E Grover, Lei Gong \\ and Jonathan F Wendel ${ }^{*}$
}

\begin{abstract}
Background: Centromeric DNA sequences alone are neither necessary nor sufficient for centromere specification. The centromere specific histone, $\mathrm{CenH}$, evolves rapidly in many species, perhaps as a coevolutionary response to rapidly evolving centromeric DNA. To gain insight into $\mathrm{CenH} 3$ evolution, we characterized patterns of nucleotide and protein diversity among diploids and allopolyploids within three diverse angiosperm genera, Brassica, Oryza, and Gossypium (cotton), with a focus on evidence for diversifying selection in the various domains of the CenH3 gene. In addition, we compare expression profiles and alternative splicing patterns for $\mathrm{CenH} 3$ in representatives of each genus.
\end{abstract}

Results: All three genera retain both duplicated CenH3 copies, while Brassica and Gossypium exhibit pronounced homoeologous expression level bias. Comparisons among genera reveal shared and unique aspects of $\mathrm{CenH} 3$ evolution, variable levels of diversifying selection in different $\mathrm{CenH} 3$ domains, and that alternative splicing contributes significantly to CenH3 diversity.

Conclusions: Since the $\mathrm{N}$ terminus is subject to diversifying selection but the DNA binding domains do not appear to be, rapidly evolving centromere sequences are unlikely to be the primary driver of $\mathrm{CenH}$ sequence diversification. At present, the functional explanation for the diversity generated by both conventional protein evolution in the $\mathrm{N}$ terminal domain, as well as alternative splicing, remains unexplained.

Keywords: Diversifying selection, CenH3 evolution, Alternative splicing, Centromeres, Gossypium, Brassica, Oryza

\section{Background}

The centromere is a specific region of the eukaryotic chromosome that is the assembly point of the kinetochore, a group of proteins that act as a tether for microtubules during cell division. Although eukaryotic centromeres have highly conserved machinery for chromosome segregation, centromere sequences and binding proteins specific to centromeric chromatin are highly variable, even among closely related taxa [1,2]. Specific retroelements and highly homogenized tandem repeats are common in the DNA of eukaryotic centromeres [3,4], though these too apparently diverge rapidly among closely related species $[2,5,6]$. Appropriate recruitment of these potentially co-evolving molecular components to the same site is enigmatic, as is their mechanism of spread and homogenization among

\footnotetext{
* Correspondence: jfw@iastate.edu

Department of Ecology, Evolution, and Organismal Biology, lowa State University, Ames, IA 50011, USA
}

(c) 2014 Masonbrink et al.; licensee BioMed Central. This is an Open Access article distributed under the terms of the Creative Commons Attribution License (http://creativecommons.org/licenses/by/4.0), which permits unrestricted use, distribution, and reproduction in any medium, provided the original work is properly credited. The Creative Commons Public Domain Dedication waiver (http://creativecommons.org/publicdomain/zero/1.0/) applies to the data made available in this article, unless otherwise stated. chromosomes. Explanations for the apparent paradox between conservation of function but variability in sequence typically invoke an interplay between centromere function, centromere sequences, and epigenetic factors, such that DNA sequences per se become less functionally constrained $[2,6,7]$. Accordingly, it is generally accepted that centromeres are specified epigenetically [4].

The evolution of $\mathrm{CenH} 3$ is of particular interest due to its centrality in centromere specification and function [8]. Unlike its highly conserved counterpart (histone $\mathrm{H} 3)$, CenH3 has extensive sequence variability, particularly in two regions: (1) the non-canonical NH2 terminal tail, (2) the longer loop 1 region $[9,10]$. DNA sequence diversity as well as alternative splicing can both play roles in generating diversity, which is thought to compensate for the fast-evolving centromeric DNA to ensure consistent centromeric function, although centromeric sequences are neither necessary nor sufficient for kinetochore assembly $[2,11]$. 
Given the twin observations of rapid sequence homogenization of centromeric repeats among chromosomes within species, yet rapid divergence among repeats between species, the question arises as to the fate of centromeric repeats, and CenH3 evolution, in allopolyploids. Polyploidy plays an integral role in the evolution of many organisms, particularly plants [12-14]. The consequences of polyploidy are often extensive [15-23], and the resulting duplicated genes have myriad possible fates $[18,24]$, including gene loss. Patterns of gene retention and loss following genome duplication have been extensively studied with respect to their broad classifications [25-29]. Because two presumably divergent suites of centromeric sequences become reunited into common nucleus at the time of allopolyploid formation, it is intriguing to investigate the subsequent evolutionary dynamics of the centromeric repeats as well as the now duplicated $\mathrm{CenH} 3$ sequences. In most modern diploids, CenH3 appears to have returned to single copy status following paleopolyploidy events [30], with a few notable exceptions [31]. In contrast, recent allopolyploids often have multiple $\mathrm{CenH}$ gene copies [30-32].

Here we evaluate the fate of $\mathrm{CenH} 3$ in allopolyploids from three divergent genera (Brassica, Oryza, and Gossypium) to address the question of whether the evolution of $\mathrm{CenH} 3$ is similar across a broad range of angiosperm taxa. The genera selected contain a diversity of allopolyploid species having either monophyletic or polyphyletic origins. The Brassica genus contains three diverse and widely cultivated diploid species (genomes designated A - C) and three allopolyploid species resulting from independent polyploidization events (BBCC, AACC, AABB) [33], while Oryza contains multiple diploid genome groups (designated $\mathrm{A}-\mathrm{G}$ ) and allopolyploids of diverse genomic origin (BBCC, CCDD, and HHJJ) [34]. Gossypium includes 45 diploid species divided into genome groups designated A - G and K [19], as well as a single, monophyletic [35] polyploid clade (AD genome) containing 6 species. Previous work on CenH3 in Brassica and Oryza allopolyploids has focused on selection in specific regions of the gene and the relative expression of the retained homoeologs [30,32]. Less is known about CenH3 and centromeric evolution in allopolyploid Gossypium. A prior study reported a centromeric gypsy-like retroelement (CRG) present in all centromeres of both the allotetraploid G. hirsutum (AD-genome) and the model progenitor D-genome diploid (G. raimondii), but absent from A-genome species [36]. Neither the sequence nor the expression of duplicated CenH3 have been evaluated.

We characterize CenH3 sequence evolution on in three phylogenetically disparate angiosperm genera containing diploids and allopolyploids, and assess patterns of molecular evolution. We address whether allopolyploids retain duplicated copies following allopolyploid formation; the dynamics of sequence evolution of the duplicated, newly co-resident sequences; and the relative expression levels of homoeologous copies. In addition to reporting on $\mathrm{CenH} 3$ sequence evolution within and between genera, we describe novel patterns of alternative splicing in CenH3.

\section{Results}

We cloned and sequenced the $\mathrm{CenH} 3$ genes from 7 diploid and 5 allotetraploid Gossypium species. The length of genomic sequences varied from 2565 to $2673 \mathrm{bp}$ in the diploids and 2654 to $2673 \mathrm{bp}$ in the polyploids (Additional file 1), although protein-coding length was consistent for all species, $492 \mathrm{bp}$. While length variation was not detected among cDNAs, we cannot account for length variation that occurs outside of our external primers in the first and last exons. The structure of $\mathrm{CenH} 3,7$ exons and 6 introns, was conserved among all polyploid species, (diploid cDNA sequences were inferred from genomic sequences). As expected, CenH3 was largely conserved across the genus, with the majority of polymorphisms occurring in introns. Gossypium exiguum exhibited the greatest difference observed among the diploids (93 bp deletion in intron 6); the remainder of the polymorphisms were small $(<10 \mathrm{bp})$, five of which were phylogenetically informative (Additional file 2).

Since CenH3 is thought to co-evolve with rapidly changing centromeric DNA, we mapped nonsynonymous substitutions to the secondary protein structure for three genera (Brassica, Oryza, and Gossypium) (Figure 1) in order to localize evolution along the protein. The $\mathrm{N}$ terminus was the only domain with high levels of nonsynonymous substitutions that was consistent between all three genera. Brassica had the highest numbers of nonsynonomous substitutions, which was followed by Oryza and then Gossypium. This observation is in agreement with previous analyses which show that rates of molecular evolution are faster in herbaceous plants (Oryza and Brassica) than in trees and shrubs (Gossypium) [37].

To determine the extent of CenH3 evolution, we compared $\mathrm{Ka} / \mathrm{Ks}$ (nonsynonymous/synonymous substitutions) ratios for all CDSs of a species from each genus with respect to an outgroup. CenH3 is indeed a relatively fast evolving gene, with the Brassica, Oryza, and Gossypium genes falling in the $81 \mathrm{st}$, 85th, and 97th percentile of all genes in these genomes, respectively (Figure 2).

To quantify CenH3 evolution in each genus we estimated the mean $\mathrm{Ka} / \mathrm{Ks}$ of $\mathrm{CenH} 3$ using DNAsp (Table 1) [38]. The overall mean $\mathrm{Ka} / \mathrm{Ks}$ ratios within each genus and between each genus and an outgroup all indicate that diversifying selection is absent from the CenH3 gene as a whole. To determine whether this finding is consistent at a finer scale, we used the mixed effects model of evolution software (MEME) [39] to evaluate selection at 


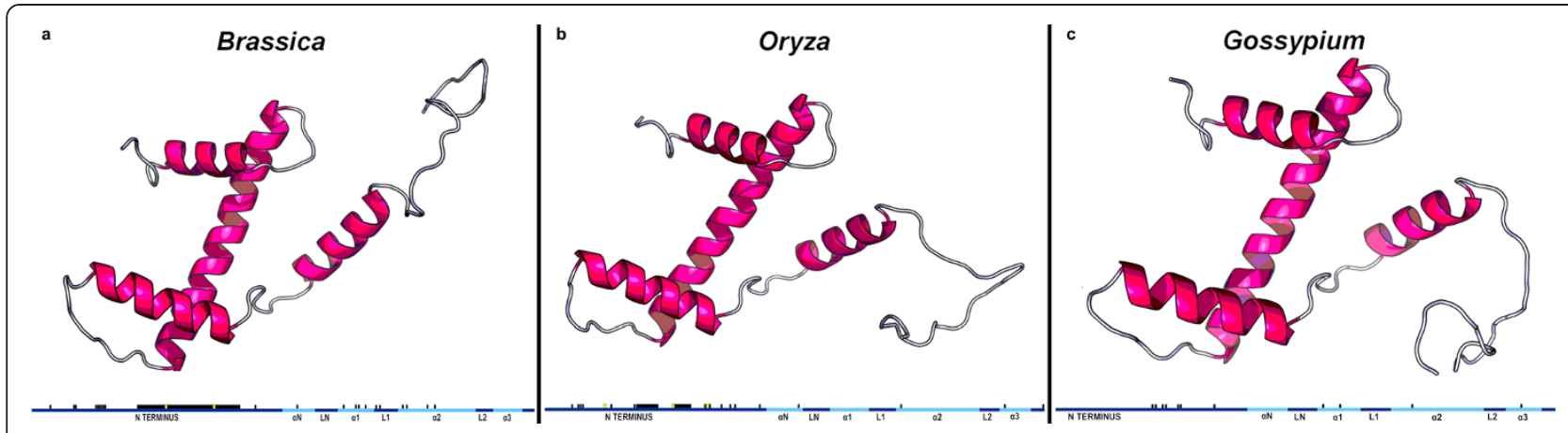

Figure 1 3D structures of CenH3 proteins from a single species in each genus. a. Brassica napus (AC8). b. Oryza australiensis isolate (EE). c. Gossypium raimondii (D5). The secondary protein structures are depicted below each 3D model containing nonsynonymous SNPs within each genus mapped to the secondary structure of the CenH3 protein. The different shades of blue signify the borders of each protein domain; a signifies an alpha helix and $L$ signifies a loop. Black rectangles signify nonsynonymous SNPs, while yellow rectangles signify nonsynonymous SNPs under positive selection (Figure 3). The $\mathrm{N}$ terminus was the primary site of length variation among the three genera and the long black rectangle in the $\mathrm{N}$ terminus of the Brassica CenH3 signifies gaps in the alignment among Brassica species.

the codon level in each genus (Table 2, Figure 3). In Brassica and Oryza, the only codons with evidence suggestive of diversifying selection were limited to the $\mathrm{N}$ terminus, while no evidence of diversifying selection was found for Gossypium. Inferred codons subject to selection in Oryza and Brassica were phylogenetically episodic and specific to only a few species for each codon (Figure 3).

\section{CenH3 evolution in allopolyploids}

Given the single copy status observed in most diploid angiosperms sequenced to date, despite an evolutionary history, which encompasses multiple episodes of polyploidy, we wished to address whether or not $\mathrm{CenH} 3$ has been retained in duplicate following recent allopolyploidy events. In Gossypium, we found that each diploid representative had a single $\mathrm{CenH} 3$ gene and that all allopolyploid species had two, indicating retention of both parental copies, as reported for Brassica and Oryza $[30,32,40]$.

To address whether or not duplicated $\mathrm{CenH} 3$ sequences evolve independently of one another following allopolyploid formation, or if instead they are subjected to some form of sequence interaction or homogenization, we manually analyzed $\mathrm{CenH} 3$ sequences from all three genera. This lack of independence has been demonstrated for other homoeologous single copy genes in allopolyploids, most notably in Gossypium where the phenomenon was first described [41,42]. CenH3 gene conversion was absent in all three genera, which is consistent with previous reports in Brassica and Oryza [30,32].

Genomic DNA sequences for CenH3 from Gossypium were used to construct a maximum likelihood tree, which concurs with the currently accepted phylogeny for the genus (Additional file 3). We resolved monophyletic clades for both the $A_{T}$ and $D_{T}$ homoeologs and their respective model diploid progenitors. Gene conversion was not detected in the homoeologs, further confirming that independent evolution of $\mathrm{CenH} 3$ homeologs occurred in this 1-2 MYD polyploid clade.

\section{CenH3 gene expression}

As mentioned above, there are various possible fates for genes duplicated via polyploidy. While both parental copies of $\mathrm{CenH} 3$ were retained in all studied allopolyploid species of Oryza, Brassica, and Gossypium, the transcriptional usage of each parental copy can vary from equivalent to complete silencing of one parental copy. To assess expression of CenH3 in Gossypium, we used three independent methods that allow us to assess the relative expression of homoeologs. The same tissue source was used for clone-counting and chromatogram measurements, while RNA-seq data from different sources was used to investigate other aspects of CenH3 expression. The RNAseq data sources included (A2 vs D5) to determine the relative expression of $\mathrm{CenH} 3$ in the model diploid progenitors to the cotton polyploids, a synthetic hybrid between these two diploids (A2XD5F1), a synthetic polyploid (2_A2D1), and a domesticated and wild accession of AD1 (maxxa and yuc respectively).

The three methods to analyze expression resulted in differing degrees of homoeolog bias (where $\mathrm{A}_{T}$ and $\mathrm{D}_{\mathrm{T}}$ are used to denote the two homoeologs), which was moderate in the RNA-seq data, and more extreme in the other methods (Figure 4). $\mathrm{A}_{\mathrm{T}}$ homoeolog, expression was favored in every species, tissue, and test (Figure 4). With RNA-seq data we compared the total expression levels of the model progenitor diploids (A2 vs D5), a synthetic polyploid (2(A2D1)), and wild and domesticated accessions of AD1 (yucatanense and Maxxa, respectively), all of which lacked a significant difference in expression. The only sample with a significant expression bias was the F1 


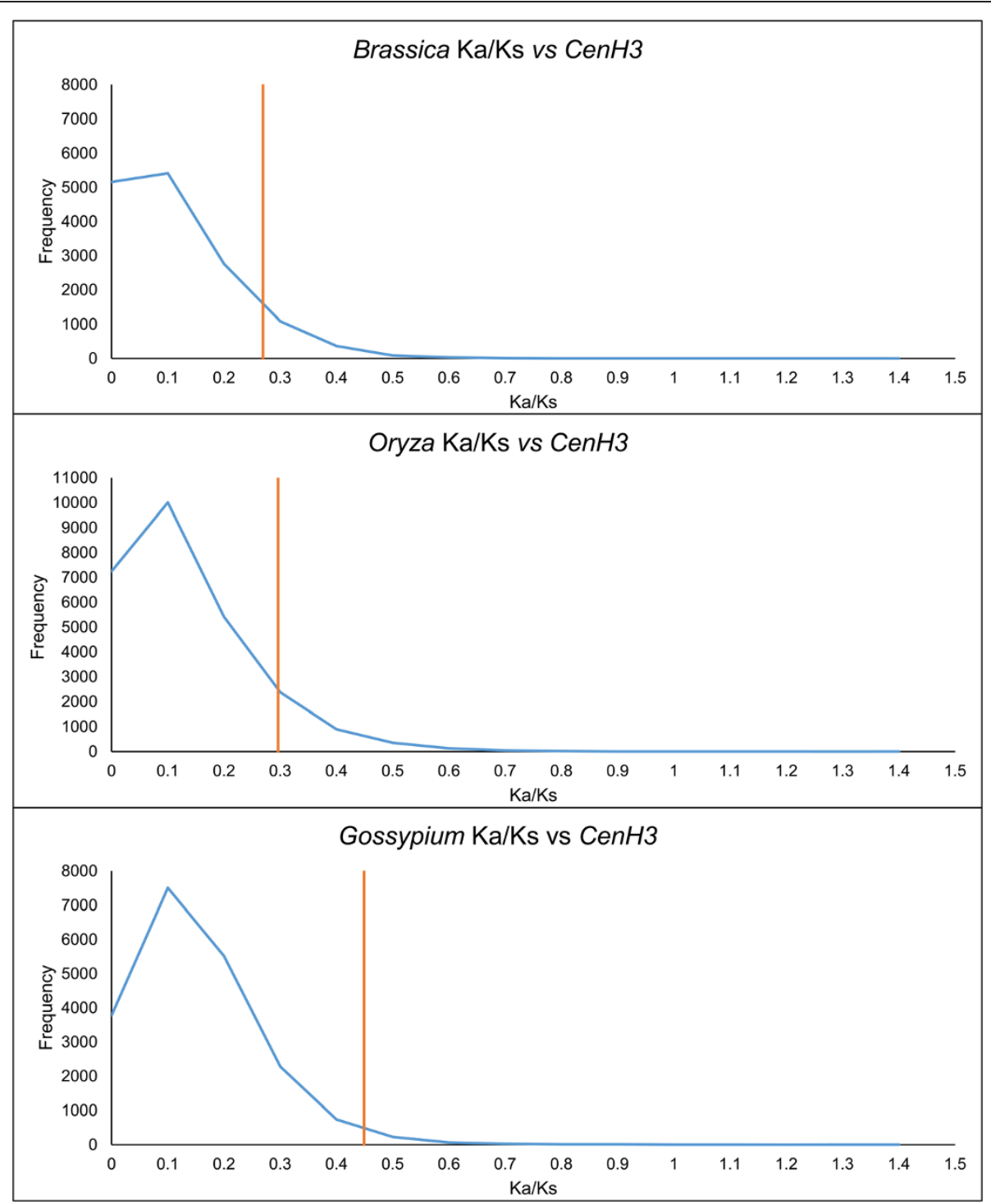

Figure 2 The $\mathrm{Ka} / \mathrm{Ks}$ ratio for the CDS from a representative species of Brassica, Oryza, and Gossypium. Each representative species was used to compare the rate of $\mathrm{CenH} 3$ evolution to other genes in each genus. The $\mathrm{y}$-axis is the number of genes that correspond to the Ka/Ks bin values on the $\mathrm{x}$-axis. The blue line is the whole genome $\mathrm{Ka} / \mathrm{Ks}$, and the orange line is $\mathrm{CenH} 3$ from each taxon.

(A2D5), biased at $87.5 \%(\mathrm{P} \leq 0.05)$. The difference in homoeolog expression was not significantly different ( $T$ test) between leaf and bud tissue, except that $A_{T}$ homoeolog expression was significantly higher in leaves for G. barbadense $\left(\mathrm{P}=1.1024 \times 10^{-10}\right)$.

Table 1 Jukes Cantor corrected estimates of $\mathrm{Ka} / \mathrm{Ks}$ within each genus, and between each genus and outgroup

\begin{tabular}{|c|c|c|c|c|c|c|}
\hline & \multicolumn{3}{|l|}{ Within } & \multicolumn{3}{|l|}{ Between } \\
\hline & Brassica & Oryza & Gossypium & Brassica & Oryza & Gossypium \\
\hline $\mathrm{Ka}$ & 0.045 & 0.029 & 0.008 & 0.143 & 0.218 & 0.025 \\
\hline Ks & 0.126 & 0.132 & 0.020 & 0.550 & 0.600 & 0.039 \\
\hline $\mathrm{Ka} / \mathrm{Ks}$ & 0.354 & 0.217 & 0.401 & 0.260 & 0.390 & 0.643 \\
\hline
\end{tabular}

Due to the sequence similarity between homoeologs, specific primers could not be designed for quantitative PCR, and thus we measured homoeolog expression bias with three separate methods, accompanied by different caveats. Due to the low number of SNPs between the homoeologs, a large number of RNA-seq reads could not be allocated to a particular homoeolog. We also cloned CenH3 cDNAs, which were counted to calculate relative expression of homoeologs, albeit with a smaller sample size (Additional file 4).

\section{Alternative splicing}

Alternative splicing of transcripts is one mechanism by which novel proteins are created, which conceivably may 
Table 2 Significant $p$-values associated with codons in Brassica and Oryza, as inferred from MEME analysis

\begin{tabular}{ll}
\hline Genus/codon & P-value \\
\hline Brassica/52 & 0.0379 \\
Brassica/69 & 0.009 \\
Oryza/34 & 0.008 \\
Oryza/45 & 0.030 \\
Oryza/57 & 0.045 \\
\hline
\end{tabular}

provide the diversity for interaction between rapidly evolving partners like CenH3 and centromeric DNA. To address how alternative splicing affects $\mathrm{CenH} 3$, we assessed the level of alternative splicing for each homoeolog of CenH3 in allopolyploid Gossypium by cloning and sequencing the amplified CenH3 cDNAs from leaf and leaf bud tissues. The sum frequency of alternatively spliced transcripts for both tissues was 26.7\% (55/206 transcripts) and consisted of 33 exon deletions and 22 intron retentions (relative to the major variant) for all allopolyploids. Three splicing variants were found in all species evaluated (Figure 5): (1) $45 \mathrm{bp}$ intron retention at position 99 (8.3\% of transcripts); (2) a 6 bp exon deletion from position 137-142 (9.2\% of transcripts); and (3) a $39 \mathrm{bp}$ exon deletion in $\mathrm{A}_{\mathrm{T}}$ homeologs from $137-175$ (3.4\% of all transcripts or $4.9 \%$ of $\mathrm{A}_{\mathrm{T}}$ transcripts; Figure 5). One splicing variant, a 12 bp intron retention, was shared only between G. hirsutum (AD1) and G. tomentosum (AD3) (Figure 5). The most common splicing variants resulted in either a slight deletion or extension of the $\mathrm{N}$ terminus, thus adding diversity to an already rapidly evolving domain of $\mathrm{CenH} 3$, while the least common splicing variants resulted in nonfunctional protein predictions.

G. hirsutum (AD1) had the highest frequency of spliced transcripts at $44.4 \%$ (Additional file 5). Each species had a different proportion of each splicing variant; for example, the exon 137-142 deletion was present in $25 \%$ of G. hirsutum (AD1) clones, while it was present at $2.9-7.8 \%$ of other species. $17.6 \%$ of G. mustelinum (AD4) clones included the $45 \mathrm{bp}$ intron insertion at position 99, which was only present $5.6-7.8 \%$ in the other species (Figure 5).

\section{Discussion}

Despite its crucial role in centromere specification, many aspects of $\mathrm{CenH} 3$ evolution are poorly understood. Recent years have brought advances in our understanding of centromere epigenetics and evolution. For example, tandem repeats in many species have an evolutionary relationship with the CenH3 protein to reduce nucleosomal bending energy [43], multiple proteins interact with centromeric DNA to induce positive supercoiling of centromeric DNA [44], and CenPA (CenH3 in plants) provides the foundation for binding other kinetochore proteins [45].

\section{CenH3 sequence evolution in angiosperms}

The present study extends our knowledge of the pace and process of $\mathrm{CenH} 3$ evolution by evaluating genomic and expression changes in three diverse angiosperm genera. Previous research in Brassica and Oryza showed that the CenH3 N terminus and CATD (loop 1 and $\alpha 2$ helix domains) sequences were under diversifying selection in lineage specific manners [30,32]. To assess the generality of these findings in angiosperms, we reanalyzed the Oryza and Brassica sequences at a finer scale to identify specific regions of $\mathrm{CenH} 3$ that have actively diversified, and performed the first $\mathrm{CenH} 3$ sequence analysis for Gossypium. Across all three genera, diversification generally occurs in the $\mathrm{N}$ terminus, a result consistent with previous reports of rapid evolution in this domain, but contrary to reports of diversification in the CenPA Targeting Domain [10,46-50].

\section{Retention and expression of $\mathrm{CenH} 3$ alleles in allopolyploids}

In all three genera, both homoeologous copies are retained following genome doubling, demonstrating that restoration to single copy status, as widely observed among modern "diploid" plants, need not occur quickly following WGD events. Interestingly, expression of homoeologous $\mathrm{CenH} 3$ copies in Gossypium exhibited directional bias in all samples, although differences were not always statistically significant (Figure 4); this result contrasts with reports from Brassica and Oryza allopolyploids [30,32]. In Brassica allotetraploids, a variety of $\mathrm{CenH} 3$ expression patterns were found for homoeologs, from a 2:1 ratio in an accession of $B$. juncea to complete B-genome CenH3 suppression in an accession of $B$. carinata [32]. In allotetraploid O. minuta and O. alta, CenH3 expression is unbiased [30]. The variation in expression profiles among allopolyploids in these three genera is notable and without an obvious explanation, although it seems likely that homoeolog expression levels reflect the unique genomic and evolutionary idiosyncrasies that characterize hybridization and genome doubling in each genus.

\section{Alternative splicing of $\mathrm{CenH} 3$}

In addition to non-synonymous evolution, protein diversity may be generated by alternative splicing. Considering the three genera collectively, it appears that alternative splicing frequently modifies the $\mathrm{N}$ terminus of $\mathrm{CenH} 3$ in Brassica and Gossypium, and this is the only domain modified in Oryza [30,32]. Interestingly, the $\mathrm{N}$ terminus often cannot be aligned among closely related genera, yet it is necessary for centromeric deposition of $\mathrm{CenH} 3$ during meiosis in $A$. thaliana [51,52]. The $\mathrm{N}$ terminus also 


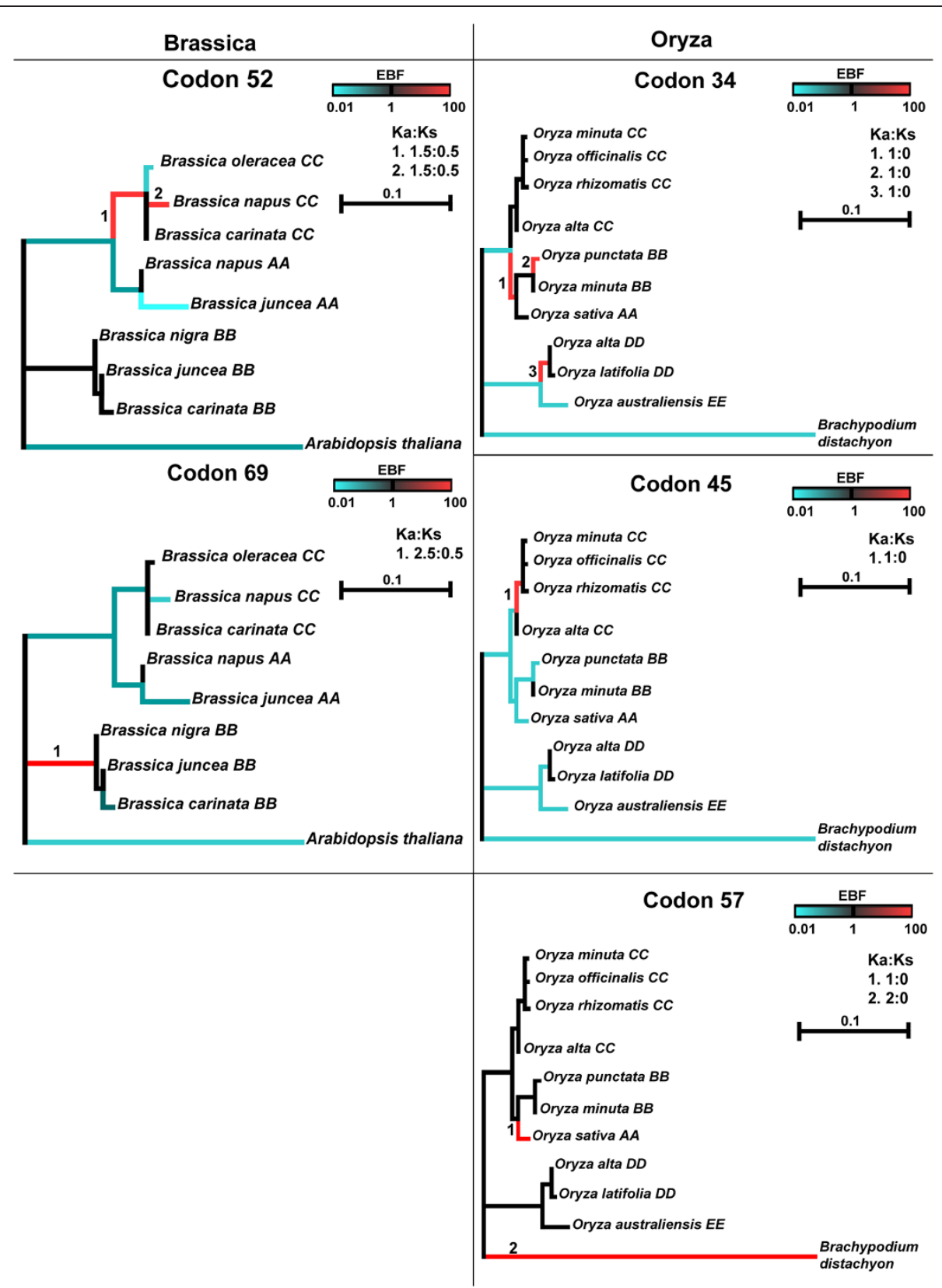

Figure 3 Episodic diversifying selection in Brassica and Oryza, as inferred using MEME. Each phylogeny represents a single codon exhibiting diversifying selection in the specified genus. The scale bar represents distance and EBF is the empirical Bayes factor, which signifies diversifying selection with warm colors and stabilizing selection with cool colors. Particular branches are labeled with numbers, which correspond to the Ka:Ks of a single codon on the right of each phylogeny.

interacts with kinetochore proteins in S. cerivisiae [53]. Perhaps alternative splicing represents another means to generate the diversity of sequences necessary for CenH3 to target centromeres in meiosis, or a rapid defense that introduces new CenH3 proteins in response to increased centromere size. An alternative is that alternative splicing can also lead to a differing abundance of alternate transcripts between cells and tissues, which has implications in centromeric DNA if CenH3 and centromeric DNA are indeed coevolving.

\section{Conclusions}

By comparing the $\mathrm{CenH} 3$ sequences from three disparate angiosperm genera, we have gained insight into the rates and regions of evolution in this important protein. The most commonly mutated domain is the $\mathrm{N}$ terminus, which 


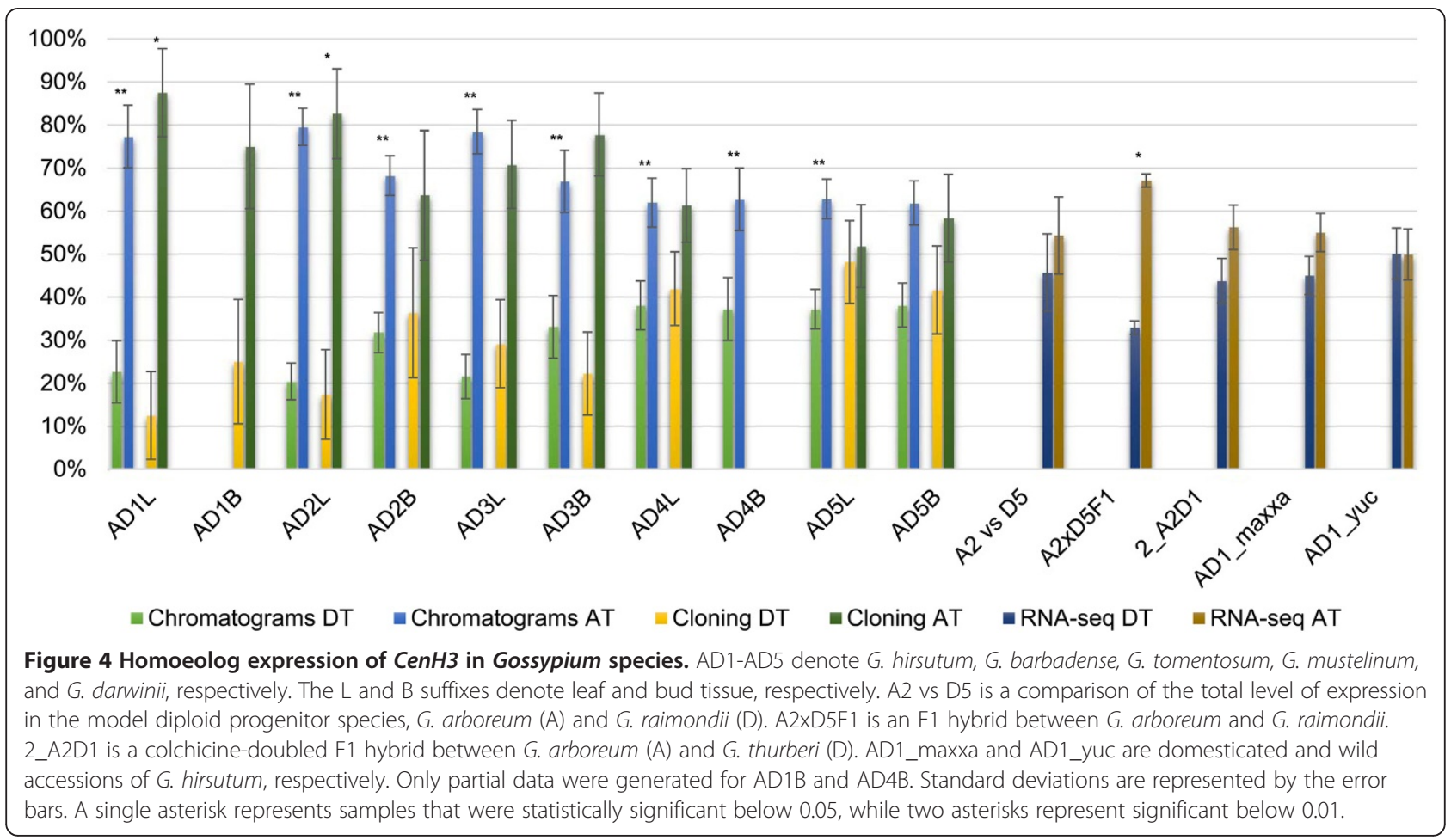

is also subject to alternative splicing, and contributes significantly to diversity in the $\mathrm{N}$ terminus of Brassica and Gossypium CenH3s. Alternative splicing is largely absent from the histone fold domain, even though loop 1 and $\alpha 2$ helix domains bind centromeric DNA, which therefore likely are subject to length constraints.
While CenH3 is considered a rapidly evolving protein, the $\mathrm{N}$ terminus is the only domain that is unalignable among closely related genera and is the most diverse domain. Roles have been attributed to the $\mathrm{N}$ terminus of $\mathrm{CenH}$, such as ubiquitin-mediated proteolysis [53] and it is indispensable for meiotic localization of $\mathrm{CenH} 3$, yet

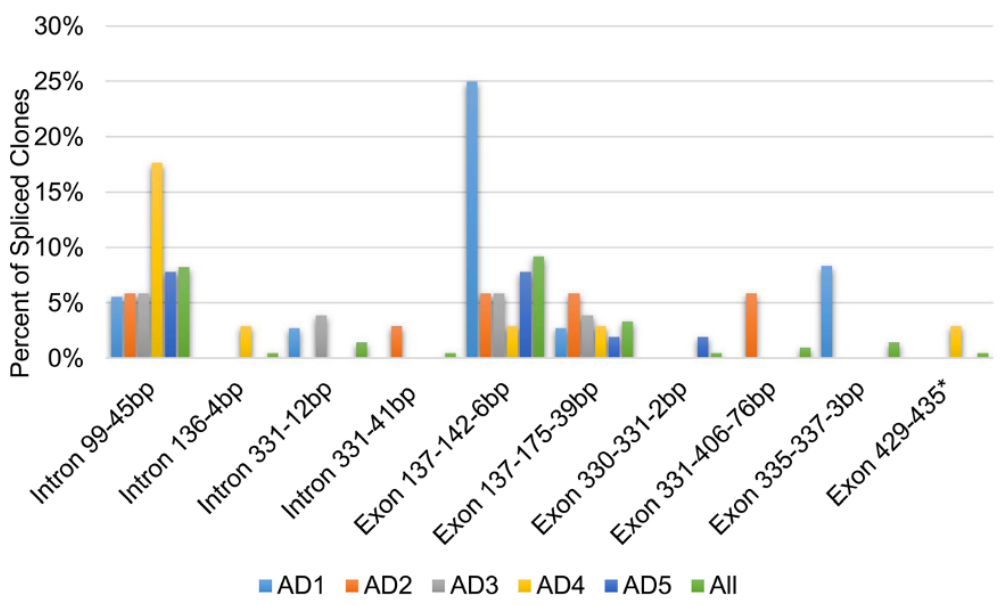

Figure 5 Frequency of splicing variants in all Gossypium allopolyploids. Splicing variants found from cloning PCR amplified CDNAs and displayed as a percent of the total number of clones obtained from each species. For the "All" category the splicing variants are displayed as a percent of the total number of clones obtained for all species combined. Exon stands for exon deletion and Intron denotes an intron retention. The asterisk on EXON 429-435 stands for unknown, since the PCR product ended at nucleotide position 435. AD1-AD5 denote different polyploid species in numerical order: G. hirsutum, G. barbadense, G. tomentosum, G. mustelinum, and G. darwinii. CenH3 protein secondary structure is aligned to the nucleotide positions of the cDNA below. 
the domain is expendable in mitosis [51,52]. In humans the $\mathrm{N}$ terminus is a hotspot for posttranslational modifications that interact with other centromeric proteins [54], and perhaps this is the case in plants as well. At present, the functional explanation for the diversity generated by both conventional protein evolution in the $\mathrm{N}$ terminal domain, as well as alternative splicing, remain unexplained.

The basis for the interest in CenH3 is its ability to bind to rapidly evolving centromeric repeats and yet still interact with conserved elements in the kinetochore. The dynamics of this relationship at the polyploid scale are increasingly complex due to the duplication of $\mathrm{CenH} 3$ and exposure to a new regime centromere repeats. The mono and polyphyletic origins of polyploids seem to influence the direction and level of expression bias between $\mathrm{CenH} 3$ homoeologs (Figure 4) and is most pronounced after hybridization, as is seen in the F1(A2D5) of Gossypium, but not following polyploidy 2(A2D1). Another compelling issue is obtaining diversity in these transcripts to respond to repeat evolution in the centromere, which is sparse in our tests for selection. Alternative splicing may be an another means to obtain this diversity, although neither selection nor alternative splicing modified the DNA binding domain of CenH3. Since the N terminus is the only domain modified by alternative splicing and subject to diversifying selection, but the DNA binding domains are not, rapidly evolving centromere sequences are unlikely to be the primary driver of $\mathrm{CenH} 3$ sequence diversification.

\section{Methods}

\section{Plant materials}

Leaves $(2-4 \mathrm{~cm})$ and leaf buds were collected from eight diploid cottons: G. arboreum cv. 101 [A2] (ISC427583), G. anomalum [B1] (ISC447893), G. robinsonii [C2] ISC451818), G. raimondii [D5] (ISC429440), G. stocksii [E1] (ISC447876), G. longicalyx [F1] (ISC418550), G. bickii [G1] (ISC414834), G. exiguum [K] (ISC416400), and from five allopolyploid cottons (G. hirsutum cv. TM1 [AD1] (ISC451819), G. barbadense cv. Pima S6 [AD2] (ISC45 1820), G. tomentosum 95 [AD3] (ISC451821), G. mustelinum local lab accession [AD4] (ISC429442), and G. darwinii PW45 [AD5] (ISC429431). For purposes of phylogenetic reconstruction, we included the outgroup species Gossypioides kirkii (ISC 418555) [55]. All lines were grown in the Pohl Conservatory at Iowa State University and were used for both DNA and RNA extractions. DNA was extracted using the Qiagen DNeasy Plant Kit following the manufacturers recommended protocol. RNA was extracted using the Sigma Spectrum RNA Extraction Kit following the manufacturers recommended protocol with the following modifications: protocol A was followed at step 4 , one wash each was performed for wash solutions I and II, and on-column DNA digestion was performed with the Sigma On-Column DNase I Digest Set.

\section{CenH3 sequencing}

$\mathrm{CenH} 3$ gene sequence data for G. raimondii was obtained from Phytozome [56,57], from which primers were designed (Additional file 6) for PCR amplification and sequencing from other species. PCR amplifications were performed using the manufacturers recommended reaction mixtures/cycling conditions and a melting temperature of $57^{\circ} \mathrm{C}$. PCR amplicons from all diploid accessions (except $G$. exiguum) were cleaned via the Qiaquick PCR column cleanup (Qiagen) and sequenced with the amplification primers and a set of internal sequencing primers (Additional file 6).

PCR products from G. exiguum and all polyploid species were visualized on an Invitrogen E-gel to isolate bands, and cloned with the P-GEM-T Easy Vector ligation kit (Promega) and Top10 Competent Cells (Invitrogen) according to the recommended protocol. Clones were sequenced at the Iowa State DNA Sequencing Facility using both M13 primers and internal primers (Additional file 6).

CenH3 cDNA sequences for Oryza and Brassica were downloaded from GenBank [58] and Phytozome [57] (Additional file 7).

\section{Evaluation of selection}

Jukes Cantor corrected estimates of $\mathrm{Ka} / \mathrm{Ks}$ were measured using DNAsp. The MEME software package [39] accessed via the (http://www.datamonkey.org) server [59], was used to test for selection at the codon level. The automatic selection tool was used to determine the correct substitution models for Brassica (F81), Gossypium (F81), and Oryza (HKY85). The significance level cutoff was set at $\mathrm{P}=<0.05$. We used Arabidopsis thaliana (24 million years divergence (MYD); Lysak et al. [60]), Gossypioides kirki (13.6 MYD; Cronn et al. [61]), and Brachypodium distachyon (46 MYD; Sanderson [62]) as the outgroups for Brassica (7.9 MYD; Jacquemin et al. [63]), Gossypium (5-10 MYD; Senchina et al. [64]), and Oryza (15 MYD; Sanderson [62]), respectively.

We used the SynMap tool of CoGe (http://genomeevolution.org/CoGe) $[65,66]$ to identify blocks of syntenic orthologs to evaluate whole genome $\mathrm{Ka} / \mathrm{Ks}$ for a representative species from each genus (Brassica rapa, Oryza sativa, Gossypium raimondi). The following parameters were used: BlastN, relative gene order, $-\mathrm{D} 50,-\mathrm{A} 10$, quota align merge $-\mathrm{Dm} 80$, quota align with a ratio of coverage depth at 3:1 for (B. rapa: A. thaliana), 1:1 for (O. sativa: $B$. distachyon), and 6:1 for (G. raimondii: Theobroma cacao), overlap distance 40. Each species was compared to their previously described outgroups, except 
T. cacao (60 MYD) [67] was the outgroup for G. raimondii, since Gossypioides kirki lacks a sequenced genome.

\section{Phylogenetic analysis}

Genomic DNA sequences were aligned using CLUSTALW [68] in BioEdit [69] and converted to NEXUS format using readal (http://trimal.cgenomics.org). The best fitting model of DNA sequence evolution was determined using the AIC and BIC, as calculated by jModelTest [70,71]. Since both the GTR $+\Gamma$ model and the $\mathrm{HKY}+\Gamma$ model were favored by AIC and BIC respectively, MEGA6 was used to build bootstrapped maximum likelihood trees with 100 replicates under both models [72,73]. The log likelihood of the GTR $+\Gamma$ tree was slightly higher and is reported here; however, both trees exhibited a similar topology.

\section{Protein structure prediction}

Secondary and tertiary protein structures were modeled using RaptorX [74-77]. This software compares alignments of the sample protein to other proteins with known structural information to determine a probable structure using statistics. A representative $\mathrm{CenH} 3$ sequence from each genus was modeled (G.raimondii (D5), Oryza australiensis (EE), and Brassica napus (AC8)) (Figure 1).

\section{cDNA generation and sequencing of $\mathrm{CenH} 3$ transcripts}

Reverse transcription was performed using the Invitrogen SuperScript III First-Strand Synthesis System Kit using oligo dT primers, and $\mathrm{CenH} 3$ was amplified from the cDNA pool using primers that were designed from the '5' and 3' outermost exons (Additional file 6). PCR products were processed using the Qiaquick PCR Cleanup columns and sequenced with the amplification primers (Additional file 6).

Chromatogram-based expression estimates were calculated as described previously [78]. At least three replicates were used for each tissue to permit standard error calculations and paired, two-tailed $\mathrm{T}$ tests were used to test for significance. Expression levels for the polyploid accessions were secondarily estimated with RNA-seq data and by cloning cDNA amplicons (as described above). The clones were randomly selected from each sample, sequenced, and then grouped by their subgenomic origin " $\mathrm{A}_{\mathrm{T}}$ " and " $\mathrm{D}_{\mathrm{T}}$ ". Since the samples should follow a binomial distribution, the null hypothesis for the rate of cloning each homeologous copy of CenH3 should be 0.5. To control for the FWER (Family-wise Error Rate) at $\alpha=0.05$, the Bonferroni correction was determine the significance.

\section{Expression estimation via RNA-seq}

To assess $\mathrm{CenH} 3$ gene expression, we analyzed previously generated leaf transcriptome data (SRA BioProject PRJNA171342) [79] for both model diploid parents, an F1 hybrid of G. arboreum (A2 genome) and G. raimondii
(D5 genome), a colchicine doubled F1 hybrid of $G$. arboreum and G. thurberi (D1 genome), and two accessions of the allopolyploid (AD genome) G. hirsutum (G. hirsutum var yucatanense, a wild accession; G. hirsutum cv Maxxa, a domesticated accession). Raw reads were trimmed with sickle (https://github.com/najoshi/sickle), and mapped to the generated $\mathrm{CenH} 3$ sequences using GSNAP (batch 4, novel splicing on) [80] in conjunction with a CenH3-specific SNP index to efficiently map sequences from different species and subgenomes. The SNP indices were manually curated from Sanger sequencing of the cDNA's and gene sequences. For sequences from the hybrid and polyploid, PolyCat [81] was used to partition $\mathrm{A}$ - and $\mathrm{D}$-genome derived reads. The significance for homeolog bias was calculated using a paired, Student's T-test with $\log 2$ transformation to ensure the normality in expression values.

\section{Availability of supporting data}

$\mathrm{CenH} 3$ gene sequence data have been submitted to GenBank. Accession numbers can be found in Additional file 1.

\section{Additional files}

Additional file 1: Genomic sequence lengths of $\mathrm{CenH} 3$ in Gossypium and Gossypioides. Gossypium $\mathrm{CenH} 3$ accession numbers and gene lengths.

Additional file 2: Phylogenetically informative alternative splicing variants in Gossypium allopolyploids. Splicing variants of Gossypium grouped informatively.

Additional file 3: Gossypium CenH3 Phylogeny. A CenH3 phylogeny of Gossypium diploids and polyploids. Phylogeny of Gossypium using genomic CenH3 sequences. The Gossypium phylogeny is in agreement with previously published phylogenies [19], excluding the polytomy seen in the AT sub-tree, which was the result of too few informative SNPs in the CenH3 gene. A is G. arboreum, B is $G$. anomalum, C is $G$. robinsonii, $D$ is $G$. raimondii, $\mathrm{E}$ is $\mathrm{G}$. stocksii, $\mathrm{F}$ is $\mathrm{G}$. longicalyx, $\mathrm{G}$ is $\mathrm{G}$. bickii, $\mathrm{K}$ is $\mathrm{G}$. exiguum [K], AD1 is G. hirsutum, AD2 is G. barbadense, AD3 is G. tomentosum, AD4 is G. mustelinum, AD5 is G. darwinii, Gk is Gossypioides kirkii. A scale bar is provided at the bottom; numbers at each node are bootstrap support values.

Additional file 4: The number of clones for each sub-genome in allopolyploid Gossypium. Counts of $\mathrm{CenH} 3$ clones allocated to each subgenome in Gossypium allopolyploids.

Additional file 5: Percentage of clones with splicing variations in each polyploid genome. The percentage of the total number of clones that contained splicing variants in each polyploid genome. Percent of splicing variants found in clones from each species. Total number of clones from each species was the sum total of the clones from bud and leaf tissue, if both were available. AD1-AD5 denote G. hirsutum, $G$. barbadense, G. tomentosum, G. mustelinum, and G. darwinii, respectively.

Additional file 6: Primer sequences for genomic and cDNA amplification. Primer sequences.

Additional file 7: Accessions downloaded from GenBank and Phytozome. Species names and accession numbers of Brassica and Oryza CenH3 sequences.

Competing interests

The authors declare that they have no competing interests. 


\section{Authors' contributions}

RM was involved in experimental design, the gene sequencing, cloning, and expression analyses, and drafted the manuscript. JG was involved in the conception of the evolutionary analysis and statistics, performed the phylogenetic analysis, and participated in drafting the manuscript. JJ cloned and sequenced many of the CenH3 genes and participated in drafting the manuscript. SRB was involved in experimental design and supervision for the CoGE and meme analysis, as well as being involved in drafting the manuscript. CG participated in the experimental design and helped draft the manuscript. LG participated in parts of the cloning, performed all of the statistical analyses, and wrote the methods for these analyses. JW participated in the experimental design, evolutionary analyses, and helped draft the manuscript. All authors read and approved the final manuscript.

\section{Acknowledgments}

The authors thank Patrick Edger for comments and suggestions with Brassica, Guanjing Hu for help with the Gossypium experimental work, and Matt Hufford for assistance with the evolutionary analyses. Research was supported by the NSF Plant Genome Program and by Cotton Incorporated.

Received: 15 August 2014 Accepted: 12 December 2014

Published online: 30 December 2014

\section{References}

1. Hirsch $C D$, Jiang J: Centromeres: sequences, structure, and biology plant genome diversity. In Plant Genome Diversity, Volume 1. Edited by Wendel JF, Greilhuber J, Dolezel J, Leitch IJ. Vienna: Springer; 2012:59-70.

2. Henikoff S, Ahmad K, Malik HS: The centromere paradox: stable inheritance with rapidly evolving DNA. Science 2001, 293(5532):1098-1102.

3. Willard HF, Waye JS: Chromosome-specific subsets of human alpha satellite DNA: Analysis of sequence divergence within and between chromosomal subsets and evidence for an ancestral pentameric repeat. J Mol Evol 1987, 25(3):207-214.

4. Jiang J, Birchler JA, Parrott WA, Kelly Dawe R: A molecular view of plant centromeres. Trends Plant Sci 2003, 8(12):570-575.

5. Melters DP, Bradnam KR, Young HA, Telis N, May MR, Ruby JG, Sebra R, Peluso P, Eid J, Rank D: Comparative analysis of tandem repeats from hundreds of species reveals unique insights into centromere evolution. Genome Biol 2013, 14(1):R10.

6. Meraldi P, McAinsh AD, Rheinbay E, Sorger PK: Phylogenetic and structural analysis of centromeric DNA and kinetochore proteins. Genome Biol 2006, 7(3):R23.

7. Cleveland DW, Mao Y, Sullivan KF: Centromeres and kinetochores: from epigenetics to mitotic checkpoint signaling. Cell 2003, 112(4):407-421.

8. Malik HS, Vermaak D, Henikoff S: Recurrent evolution of DNA-binding motifs in the Drosophila centromeric histone. Proc Natl Acad Sci U S A 2002, 99(3):1449-1454.

9. Talbert PB, Masuelli R, Tyagi AP, Comai L, Henikoff S: Centromeric localization and adaptive evolution of an Arabidopsis histone $\mathrm{H} 3$ variant. Plant Cell 2002, 14(5):1053-1066.

10. Malik HS, Henikoff S: Adaptive evolution of CID, a centromere-specific histone in Drosophila. Genetics 2001, 157(3):1293-1298.

11. Allshire RC, Karpen GH: Epigenetic regulation of centromeric chromatin: old dogs, new tricks? Nat Rev Genet 2008, 9(12):923-937.

12. Song C, Liu S, Xiao J, He W, Zhou Y, Qin Q, Zhang C, Liu Y: Polyploid organisms. Sci China Life Sci 2012, 55(4):301-311.

13. Heslop-Harrison JS: Genome evolution: extinction, continuation or explosion? Curr Opin Plant Biol 2012, 15(2):115-121.

14. Van de Peer $Y$, Maere $S$, Meyer A: The evolutionary significance of ancient genome duplications. Nat Rev Genet 2009, 10(10):725-732.

15. Wendel JF: Genome evolution in polyploids. Plant Mol Biol 2000, 42(1):225-249.

16. Soltis DE, Albert VA, Leebens-Mack J, Bell CD, Paterson AH, Zheng C, Sankoff D, de Pamphilis CW, Wall PK, Soltis PS: Polyploidy and angiosperm diversification. Am J Bot 2009, 96(1):336-348.

17. Adams KL, Wendel JF: Polyploidy and genome evolution in plants. Curr Opin Plant Biol 2005, 8(2):135-141.

18. Adams KL, Cronn R, Percifield R, Wendel JF: Genes duplicated by polyploidy show unequal contributions to the transcriptome and organ-specific reciprocal silencing. Proc Natl Acad Sci U S A 2003, 100(8):4649-4654.
19. Wendel JF, Flagel LE, Adams KL: Jeans, genes, and genomes: cotton as a model for studying polyploidy. In Polyploidy and Genome Evolution. Edited by Soltis PS, Soltis DE. New York: Springer; 2012:181-207.

20. Wendel JF, Doyle JJ: Polyploidy and evolution in plants. Curr Opin Plant Biol 2005, 8:135-141.

21. Soltis DE, Soltis PS: Polyploidy: recurrent formation and genome evolution. Trends Eco Evol 1999, 14(9):348-352.

22. Chen ZJ, Ni Z: Mechanisms of genomic rearrangements and gene expression changes in plant polyploids. Bioessays 2006, 28(3):240-252.

23. Chen ZJ: Genetic and epigenetic mechanisms for gene expression and phenotypic variation in plant polyploids. Annu Rev Plant Biol 2007, 58:377-406

24. Adams KL, Wendel JF: Novel patterns of gene expression in polyploid plants. Trends Genet 2005, 21(10):539-543.

25. Scannell DR, Byrne KP, Gordon JL, Wong S, Wolfe KH: Multiple rounds of speciation associated with reciprocal gene loss in polyploid yeasts. Nature 2006, 440(7082):341-345.

26. Town CD, Cheung F, Maiti R, Crabtree J, Haas BJ, Wortman JR, Hine EE, Althoff $R$, Arbogast TS, Tallon L: Comparative genomics of Brassica oleracea and Arabidopsis thaliana reveal gene loss, fragmentation, and dispersal after polyploidy. Plant Cell Online 2006, 18(6):1348-1359.

27. Ku H-M, Vision T, Liu J, Tanksley SD: Comparing sequenced segments of the tomato and Arabidopsis genomes: large-scale duplication followed by selective gene loss creates a network of synteny. Proc Natl Acad Sci U S A 2000, 97(16):9121-9126.

28. Doyle JJ, Flagel LE, Paterson AH, Rapp RA, Soltis DE, Soltis PS, Wendel JF: Evolutionary genetics of genome merger and doubling in plants. Ann Rev Genet 2008, 42(1):443-461.

29. Barker MS, Kane NC, Matvienko M, Kozik A, Michelmore RW, Knapp SJ, Rieseberg LH: Multiple paleopolyploidizations during the evolution of the Compositae reveal parallel patterns of duplicate gene retention after millions of years. Mol Biol Evol 2008, 25(11):2445-2455.

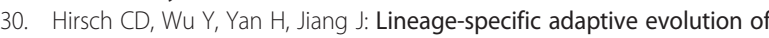
the centromeric protein $\mathrm{CenH} 3$ in diploid and allotetraploid Oryza species. Mol Biol Evol 2009, 26(12):2877-2885.

31. Lermontova I, Schubert I: $\mathrm{CenH} 3$ for establishing and maintaining centromeres. In: Plant Centromere Biology. Oxford, UK: Wiley-Blackwell; 2013: 67-82.

32. Wang G, He Q, Liu F, Cheng Z, Talbert P, Jin W: Characterization of CenH3 proteins and centromere-associated DNA sequences in diploid and allotetraploid Brassica species. Chromosoma 2011, 120(4):353-365.

33. U. N: Genome analysis in Brassica with special reference to the experimental formation of $B$. napus and peculiar mode of fertilization. Japan J Bot 1935, 7:389-452.

34. Ge S, Sang T, Lu B-R, Hong D-Y: Phylogeny of rice genomes with emphasis on origins of allotetraploid species. Proc Natl Acad Sci U S A 1999, 96(25):14400-14405.

35. Grover C, Grupp K, Wanzek R, Wendel J: Assessing the monophyly of polyploid Gossypium species. Plant Syst Evol 2012, 298(6):1177-1183.

36. Luo S, Mach J, Abramson B, Ramirez R, Schurr R, Barone P, Copenhaver G, Folkerts O: The cotton centromere contains a Ty3-gypsy-like LTR retroelement. PLoS One 2012, 7(4):e35261.

37. Smith SA, Donoghue MJ: Rates of molecular evolution are linked to life history in flowering plants. Science 2008, 322(5898):86-89.

38. Librado P, Rozas J: DnaSP v5: a software for comprehensive analysis of DNA polymorphism data. Bioinformatics 2009, 25(11):1451-1452.

39. Murrell B, Wertheim JO, Moola S, Weighill T, Scheffler K, Pond SLK: Detecting individual sites subject to episodic diversifying selection. PLoS Genet 2012, 8(7):e1002764.

40. Hui L, Lu L, Heng Y, Qin R, Xing Y, Jin W: Expression of CENH3 alleles in synthesized allopolyploid Oryza species. J Genet Genomics 2010, 37(10):703-711.

41. Salmon A, Flagel L, Ying B, Udall JA, Wendel JF: Homoeologous nonreciprocal recombination in polyploid cotton. New Phytol 2010, 186(1):123-134.

42. Flagel L, Wendel J, Udall J: Duplicate gene evolution, homoeologous recombination, and transcriptome characterization in allopolyploid cotton. BMC Genomics 2012, 13(1):302

43. Zhang T, Talbert PB, Zhang W, Wu Y, Yang Z, Henikoff JG, Henikoff S, Jiang $\mathrm{J}$ : The CentO satellite confers translational and rotational phasing on CenH3 nucleosomes in rice centromeres. Proc Natl Acad Sci U S A 2013, 110(50):E4875-E4883. 
44. Takeuchi K, Nishino T, Mayanagi K, Horikoshi N, Osakabe A, Tachiwana H, Hori T, Kurumizaka H, Fukagawa T: The centromeric nucleosome-like CENP-T-W-S-X complex induces positive supercoils into DNA. Nucleic Acids Res 2014, 42(3):1644-1655.

45. Carroll CW, Milks KJ, Straight AF: Dual recognition of CENP-A nucleosomes is required for centromere assembly. J Cell Biol 2010, 189(7):1143-1155.

46. Vermaak D, Hayden HS, Henikoff S: Centromere targeting element within the histone fold domain of Cid. Mol Cell Biol 2002, 22(21):7553-7561.

47. Cooper $\mathrm{L}$, Henikoff S: Adaptive evolution of the histone fold domain in centromeric histones. Mol Biol Evol 2004, 21(9):1712-1718.

48. Shelby RD, Vafa O, Sullivan KF: Assembly of CENP-A into centromeric chromatin requires a cooperative array of nucleosomal DNA contact sites. J Cell Biol 1997, 136(3):501-513.

49. Lermontova I, Schubert V, Fuchs J, Klatte S, Macas J, Schubert I: Loading of Arabidopsis centromeric histone CENH3 occurs mainly during G2 and requires the presence of the histone fold domain. Plant Cell 2006, 18(10):2443-2451.

50. Black BE, Foltz DR, Chakravarthy S, Luger K, Woods VL, Cleveland DW: Structural determinants for generating centromeric chromatin. Nature 2004, 430(6999):578-582

51. Lermontova I, Koroleva O, Rutten T, Fuchs J, Schubert V, Moraes I, Koszegi D, Schubert I: Knockdown of $\mathrm{CenH} 3$ in Arabidopsis reduces mitotic divisions and causes sterility by disturbed meiotic chromosome segregation. Plant J 2011, 68(1):40-50.

52. Ravi M, Shibata F, Ramahi JS, Nagaki K, Chen C, Murata M, Chan SW: Meiosis-specific loading of the centromere-specific histone $\mathrm{CENH} 3$ in Arabidopsis thaliana. PLoS Genet 2011, 7(6):e1002121.

53. Chen Y, Baker RE, Keith KC, Harris K, Stoler S, Fitzgerald-Hayes M: The N terminus of the centromere $\mathrm{H} 3$-like protein $\mathrm{Cse} 4 \mathrm{p}$ performs an essential function distinct from that of the histone fold domain. Mol Cell Biol 2000, 20(18):7037-7048.

54. Bailey AO, Panchenko T, Sathyan KM, Petkowski JJ, Pai P-J, Bai DL, Russell DH, Macara IG, Shabanowitz J, Hunt DF, Black BE, Foltz DR: Posttranslational modification of CENP-A influences the conformation of centromeric chromatin. Proc Natl Acad Sci U S A 2013, 110(29):11827-11832.

55. Seelanan T, Schnabel A, Wendel JF: Congruence and consensus in the cotton tribe. Sys Bot 1997, 22:259-290.

56. Wang K, Wang Z, Li F, Ye W, Wang J, Song G, Yue Z, Cong L, Shang H, Zhu S: The draft genome of a diploid cotton Gossypium raimondii. Nat Genet 2012, 44(10):1098-1103.

57. Goodstein DM, Shu S, Howson R, Neupane R, Hayes RD, Fazo J, Mitros T, Dirks W, Hellsten U, Putnam N: Phytozome: a comparative platform for green plant genomics. Nucleic Acids Res 2012, 40(D1):D1178-D1186

58. Benson DA, Karsch-Mizrachi I, Lipman DJ, Ostell J, Wheeler DL: GenBank. Nucleic Acids Res 2008, 36(suppl 1):D25-D30.

59. Delport W, Poon AF, Frost SD, Pond SLK: Datamonkey 2010: a suite of phylogenetic analysis tools for evolutionary biology. Bioinformatics 2010, 26(19):2455-2457.

60. Lysak MA, Koch MA, Pecinka A, Schubert I: Chromosome triplication found across the tribe Brassiceae. Genome Res 2005, 15(4):516-525.

61. Cronn RC, Small RL, Haselkorn T, Wendel JF: Rapid diversification of the cotton genus (Gossypium: Malvaceae) revealed by analysis of sixteen nuclear and chloroplast genes. Am J Botany 2002, 89(4):707-725.

62. Sanderson M: A nonparametric approach to estimating divergence times in the absence of rate constancy. Mol Biol Evol 1997, 14(12):1218.

63. Jacquemin J, Ammiraju JSS, Haberer G, Billheimer DD, Yu Y, Liu LC, Rivera LF, Mayer K, Chen M, Wing RA: Fifteen million years of evolution in the Oryza genus shows extensive gene family expansion. Mol Plant 2013, 7(4):642-656.

64. Senchina DS, Alvarez I, Cronn RC, Liu B, Rong J, Noyes RD, Paterson AH, Wing RA, Wilkins TA, Wendel JF: Rate variation among nuclear genes and the age of polyploidy in Gossypium. Mol Biol Evol 2003, 20(4):633-643.

65. Lyons E, Freeling M: How to usefully compare homologous plant genes and chromosomes as DNA sequences. Plant J 2008, 53(4):661-673.

66. Lyons E, Pedersen B, Kane J, Alam M, Ming R, Tang H, Wang X, Bowers J, Paterson A, Lisch D: Finding and comparing syntenic regions among Arabidopsis and the outgroups papaya, poplar, and grape: CoGe with rosids. Plant Physiol 2008, 148(4):1772-1781.

67. Carvalho MR, Herrera FA, Jaramillo CA, Wing SL, Callejas R: Paleocene Malvaceae from northern South America and their biogeographical implications. Am J Bot 2011, 98(8):1337-1355.
68. Thompson JD, Higgins DG, Gibson TJ: CLUSTAL W: improving the sensitivity of progressive multiple sequence alignment through sequence weighting, position-specific gap penalties and weight matrix choice. Nucleic Acids Res 1994, 22(22):4673-4680.

69. Hall TA: BioEdit: a user-friendly biological sequence alignment editor and analysis program for Windows 95/98/NT. In Nucleic Acids Symposium Series. 1999:95-98.

70. Darriba D, Taboada GL, Doallo R, Posada D: jModelTest 2: more models, new heuristics and parallel computing. Nat Methods 2012, 9(8):772.

71. Guindon S, Gascuel O: A simple, fast, and accurate algorithm to estimate large phylogenies by maximum likelihood. Syst Biol 2003, 52(5):696-704.

72. Kumar S, Nei M, Dudley J, Tamura K: MEGA: a biologist-centric software for evolutionary analysis of DNA and protein sequences. Brief Bioinform 2008, 9(4):299-306.

73. Tamura K, Stecher G, Peterson D, Filipski A, Kumar S: MEGA6: molecular evolutionary genetics analysis version 6.0. Mol Biol Evol 2013, 30(12):2725-2729.

74. Ma J, Wing RA, Bennetzen $J$, Jackson SA: Plant centromere organization: a dynamic structure with conserved functions. Trends Genet 2007, 23(3):134-139.

75. Källberg M, Wang H, Wang S, Peng J, Wang Z, Lu H, Xu J: Template-based protein structure modeling using the RaptorX web server. Nat Protocols 2012, 7(8):1511-1522.

76. Peng J, Xu J: A multiple-template approach to protein threading. Proteins 2011, 79(6):1930-1939.

77. Peng J, Xu J: Raptorx: exploiting structure information for protein alignment by statistical inference. Proteins 2011, 79(S10):161-171.

78. Adams KL, Percifield R, Wendel JF: Organ-specific silencing of duplicated genes in a newly synthesized cotton allotetraploidd. Genetics 2004, 168(4):2217-2226.

79. Yoo MJ, Szadkowski E, Wendel JF: Homoeolog expression bias and expression level dominance in allopolyploid cotton. Heredity 2013, 110(2):171-180.

80. Wu TD, Nacu S: Fast and SNP-tolerant detection of complex variants and splicing in short reads. Bioinformatics 2010, 26(7):873-881.

81. Page JT, Gingle AR, Udall JA: PolyCat: a resource for genome categorization of sequencing reads from allopolyploid organisms. G3 2013, 3(3):517-525.

\section{Submit your next manuscript to BioMed Central and take full advantage of:}

- Convenient online submission

- Thorough peer review

- No space constraints or color figure charges

- Immediate publication on acceptance

- Inclusion in PubMed, CAS, Scopus and Google Scholar

- Research which is freely available for redistribution 